\title{
Structural and magnetic properties of the colossal magnetoresistance perovskite $\mathrm{La}_{0.85} \mathrm{Ca}_{0.15} \mathrm{MnO}_{3}$
}

\author{
M. V. Lobanov \\ Department of Chemistry, Moscow State University, Moscow 119899, Russia \\ A. M. Balagurov \\ Frank Laboratory of Neutron Physics, JINR, 141980 Dubna, Russia \\ V. Ju. Pomjakushin \\ Frank Laboratory of Neutron Physics, JINR, 141980 Dubna, Russia \\ and PSI, CH-5232 Villigen, Switzerland \\ P. Fischer and M. Gutmann \\ PSI, CH-5232 Villigen, Switzerland
}

A. M. Abakumov

Department of Chemistry, Moscow State University, Moscow 119899, Russia

and EMAT, University of Antwerp (RUCA), Groenenborgerlaan 171, B-2020 Antwerp, Belgium

O. G. D’yachenko and E. V. Antipov

Department of Chemistry, Moscow State University, Moscow 119899, Russia

O. I. Lebedev* and G. Van Tendeloo ${ }^{\dagger}$

EMAT, University of Antwerp (RUCA), Groenenborgerlaan 171, B-2020 Antwerp, Belgium

(Received 19 August 1999)

\begin{abstract}
A single phase sample of $\mathrm{La}_{0.85} \mathrm{Ca}_{0.15} \mathrm{MnO}_{3}$ with uniform cation distribution and complete oxygen stoichiometry was synthesized by freeze-drying technique and studied by a variety of methods. The sample is ferromagnetic below $T_{C}=170 \mathrm{~K}$ with $\mu_{\mathrm{Mn}}=2.9(1) \mu_{B}$ at $10 \mathrm{~K}$. Precise examination of the crystal structure by a combination of electron diffraction, high-resolution electron microscopy, and neutron powder diffraction revealed a monoclinic distortion of the $\mathrm{GdFeO}_{3}$-type structure [S.G. $P 2_{1} / c, a=7.74476(6) \AA, b$ $=5.50398(4) \AA, c=5.47351(4) \AA, \beta=90.091(2)^{0}$ ] with nonequivalent $\mathrm{MnO}_{2}$ layers alternating along the $a$ axis. The crystal structure is characterized by a specific pattern of $\mathrm{Mn}-\mathrm{O}$ distances implying an unconventional orbital ordering type. A possible relation to charge ordering is described within the bond valence sum framework.
\end{abstract}

\section{INTRODUCTION}

The interest in the perovskites of the $\mathrm{La}_{1-x} \mathrm{Ca}_{x} \mathrm{MnO}_{3}$ family has been renewed in connection with the discovery of colossal magnetoresistance (CMR). ${ }^{1}$ The magnetic structure of these compounds (for a Ca content below the charge ordering point $x=0.5$ ) evolves from antiferromagnetic (AFM) for undoped $\mathrm{LaMnO}_{3}$ to ferromagnetic (FM) for high Ca concentration, although there exists a quantitative disagreement between published phase diagrams. According to the first diagram which was published by Goodenough, ${ }^{2}$ this compound is insulating and antiferromagnetic for $x \leqslant 0.2$; Schiffer et $a l .{ }^{3}$ have found that $x \approx 0.17-0.19$ is the boundary between FM-insulating (low $x$ ) and FM-metallic (high $x$ ) states, while in Huang et al. ${ }^{4}$ it is stated that in the entire $0.1<x<0.5$ region the magnetic structure of $\mathrm{La}_{1-x} \mathrm{Ca}_{x} \mathrm{MnO}_{3}$ is purely ferromagnetic and the resistivity is low. It was also stressed in this paper that the observed discrepancies are probably due to different synthesis conditions. Variation of the latter affects the stoichiometry of the compound; deviations from the ideal one (due to formation of cation or anion vacancies) change the charge carrier concentration, thus altering the magnetic state through double exchange. ${ }^{5}$ Moreover, conventional ceramic technique traditionally applied for the complex oxide preparation often suffers from insufficient homogeneity of cation distribution resulting in a variation of composition for the different crystallites within the bulk sample. Incorrect $T_{c}$ determination caused by such effects may also account for the discrepancies between $T_{c}$ values reported for the same average composition. There is also a certain ambiguity in the interpretation of the magnetic state at the crossover region $(x=0.1-0.2)$ including the possibility of phase separation. ${ }^{6}$

The CMR perovskites also exhibit a large variety of phase transformations and lattice distortions determined by several structural factors including the average size of the $A$ cations and the formal Mn valence. The $A$-cation size influences the tilting distortion of the octahedral perovskite framework whereas the Jahn-Teller effect, typical for the $\mathrm{Mn}^{+3}$ cation, leads to the imperfection of $\mathrm{MnO}_{6}$ octahedron itself. Both effects superimpose and lead to a complicated behavior of the crystal structure upon varying the chemical composition 


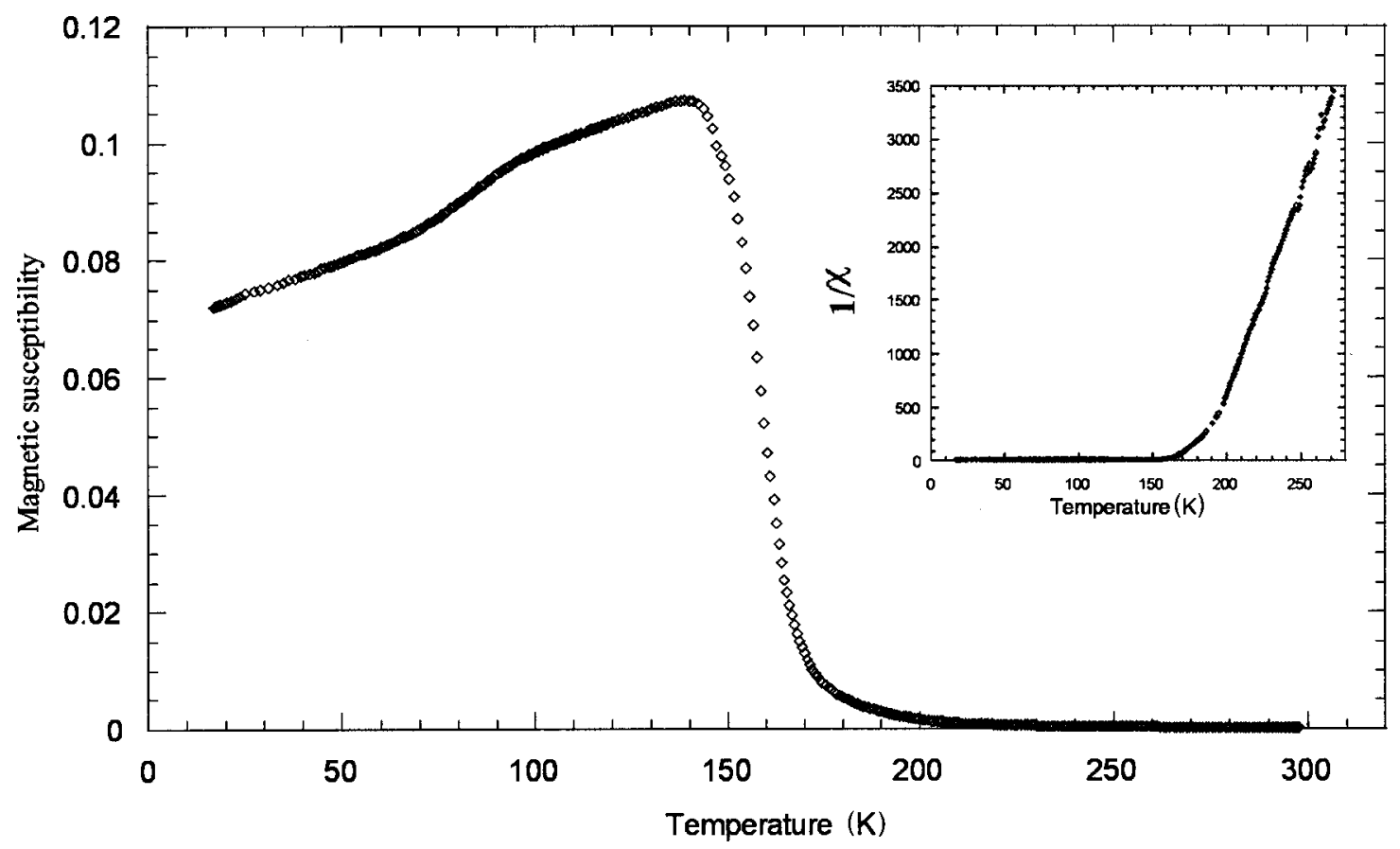

FIG. 1. Temperature dependence of magnetic susceptibility for $\mathrm{La}_{0.85} \mathrm{Ca}_{0.15} \mathrm{MnO}_{3}$ sample. Fit of the data to Curie-Weiss law is shown in the inset.

and the synthesis conditions. According to the standard structural phase diagram, ${ }^{3}$ compositions belonging to the crossover region are within the stability range of the orthorhombic (space group Pnma) structure.

Sr-containing compositions corresponding to the crossover region have recently been studied in detail. It was shown by the use of single crystal neutron and x-ray synchrotron diffraction for the particular composition $\mathrm{La}_{0.88} \mathrm{Sr}_{0.12} \mathrm{MnO}_{3}$ that a sequence of temperature-induced structural transformations ${ }^{7}$ occurs. A specific superstructure appearing at low temperature ${ }^{8,9}$ was attributed to the formation of a polaron ordered phase with relation to transport properties (resistivity minimum well below $T_{c}$ ). Recently Cox et al. ${ }^{10}$ showed that a phase separation into two phases occurs below $350 \mathrm{~K}$ with splitting of some majority-phase reflections indicative of a monoclinic $\left(\beta \approx 90.1^{\circ}\right)$ distortion of the parent orthorhombic structure. Several recent HREM observations $^{11,12}$ indicate a breakdown of orthorhombic symmetry for Ca-containing compositions, at least on the microdomain scale. Similar complications arise also in the Ca-rich region of the $\mathrm{La}_{1-x} \mathrm{Ca}_{x} \mathrm{MnO}_{3}$ system, where a stripelike contrast with $\sim 16.5 \AA$ periodicity was observed by HREM together with superstructure reflections on the electron diffraction patterns ${ }^{13,14}$ for the $x=2 / 3$ composition. These observations led to the tripling of one of the in-plane lattice parameters, and it was shown in a recent work of Radaelli et $a l .{ }^{15}$ with the use of $\mathrm{x}$-ray synchrotron data that the crystallographic superstructure can be described within a socalled "Wigner crystal" model.

We therefore attempted to prepare a stoichiometric compound with composition $\mathrm{La}_{0.85} \mathrm{Ca}_{0.15} \mathrm{MnO}_{3}$ belonging to the crossover region in order to clarify its structural and magnetic properties.

\section{SAMPLE PREPARATION AND CHARACTERIZATION}

To avoid possible inhomogeneous cation distribution and also to reduce the synthesis temperature to avoid anion va- cancy formation we applied a freeze-drying technique which is characterized by atomic-scale homogenization. Stoichiometric mixtures of titrated aqueous solutions of Mn acetate and $\mathrm{La}$ and $\mathrm{Ca}$ nitrates were sprayed into liquid nitrogen, dehydrated by sublimation (SMH-15 “Usifroid”' equipment) and then decomposed by slow heating in air. Then the mixtures were annealed in air at $900{ }^{\circ} \mathrm{C}$ for $40 \mathrm{~h}$. Single phase composition of the sample was confirmed by $\mathrm{x}$-ray powder diffraction. The $\mathrm{x}$-ray pattern of the $\mathrm{La}_{0.85} \mathrm{Ca}_{0.15} \mathrm{MnO}_{3}$ sample exhibits an orthorhombic distortion of the perovskite cell $\left(a \approx a_{\mathrm{per}} \sqrt{2}, b \approx 2 a_{\mathrm{per}}\right.$, and $\left.c \approx a_{\mathrm{per}} \sqrt{2}\right)$.

Quantitative energy-dispersive X-ray analysis (EDX) was performed with a CAMEBAX-Microbeam system. The measurements were made for 15 crystallites on the $K_{\alpha}$ peaks of $\mathrm{Mn}, \mathrm{Ca}$, and on the $L_{\alpha}$ peak of La. The cation composition inferred from EDX corresponds to the $\mathrm{La}: \mathrm{Ca}: \mathrm{Mn}$ ratio 0.84(2):0.149(3):1.00(1), in good agreement with the nominal cation stoichiometry. The ratio is virtually identical for different crystallites; numbers in parentheses are calculated statistical errors; their magnitudes indicate a highly uniform cation distribution.

The determination of the average Mn oxidation state was performed by iodometric titration. ${ }^{16}$ About $70 \mathrm{mg}$ of the sample was dissolved in concentrated $\mathrm{HCl}$. The released $\mathrm{Cl}_{2}$ was transferred by Ar flow to react with KI solution. $I_{2}$ was titrated by a standard $\mathrm{Na}_{2} \mathrm{~S}_{2} \mathrm{O}_{3}$ solution with starch added as an indicator. The analysis revealed $15 \pm 5 \%$ of $\mathrm{Mn}^{4+}$ corresponding to the oxygen index 3.00(3). Thus, combination of EDX and chemical analysis confirms the complete stoichiometry of the compound and the absence of significant deviations from the nominal composition.

Temperature dependence of the magnetic susceptibility was measured in the ac mode $\left(H_{\max }=10 \mathrm{Oe}, \omega=27 \mathrm{~Hz}\right)$ down to $12 \mathrm{~K}$. Ferromagnetic transition was detected at approximately $170 \mathrm{~K}$. For resistivity measurements a conven- 


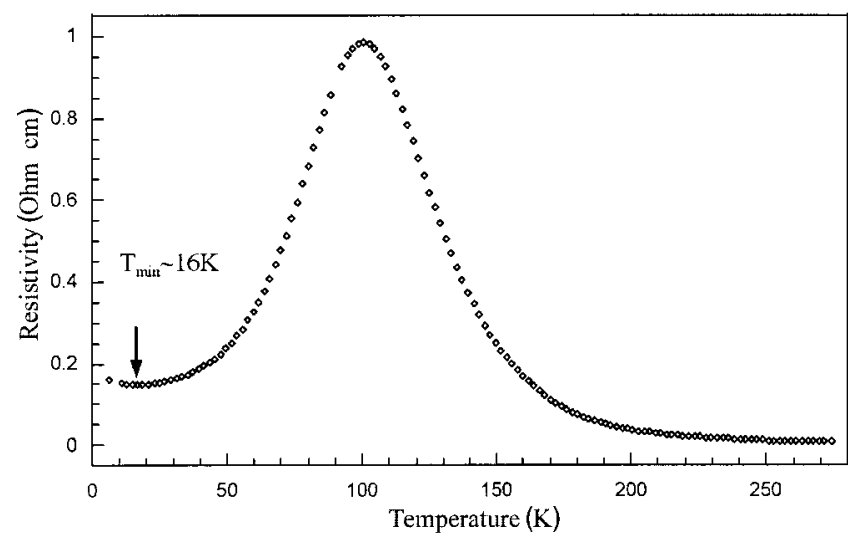

FIG. 2. Temperature dependence of electrical resistivity for $\mathrm{La}_{0.85} \mathrm{Ca}_{0.15} \mathrm{MnO}_{3}$ sample. The position of low-temperature minimum is indicated by an arrow.

tional four-probe ac- method $(\omega=32 \mathrm{~Hz})$ was applied. The electrical resistivity vs temperature curve has a typical maximum indicative of the onset of a metallic state and an upturn at about $13 \mathrm{~K}$. Negative magnetoresistance was also detected near the resistivity maximum. Temperature dependencies of magnetic susceptibility and electrical resistivity are shown in Figs. 1 and 2, respectively.

\section{MAGNETIC STRUCTURE-NEUTRON DIFFRACTION DATA}

Neutron data for magnetic structure refinement were collected with the powder diffractometer DMC $[d$-spacing range $(1.7-14.7) \AA, \delta d / d \approx 1 \%]$ at the SINQ spallation source at PSI in Villigen, using a neutron beam with wavelength $\lambda$ $=2.5616 \AA$. Figure 3 shows typical diffraction patterns at a temperature just above $T_{c}$ and at $25 \mathrm{~K}$. A ferromagnetic contribution is clearly observed at low temperature. No additional antiferromagnetic peaks were detected at any temperature. Rietveld refinement was carried out using the FULLPROF program over the scattering angle range $2 \theta=19-98^{\circ}$, corresponding to the $d$-spacing range (1.7-7.7) $\AA$. The atomic positions obtained from the high-resolution neutron diffraction data for the Pnma space group (see Table I) were fixed in the refinement of the DMC data. A ferromagnetic transition was revealed below $T_{c}=170 \mathrm{~K}$ as an increase of the

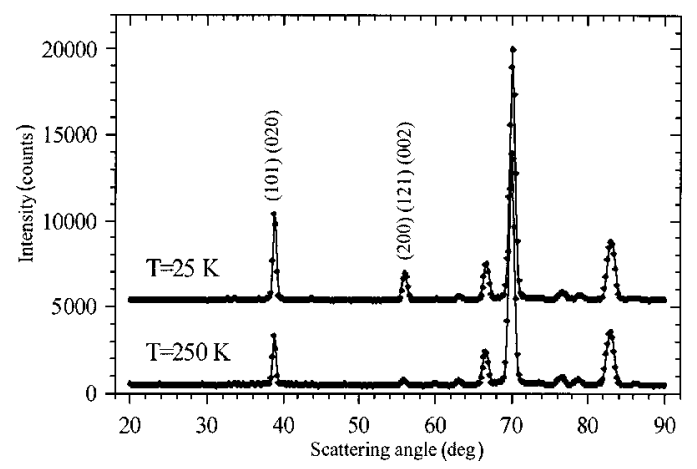

FIG. 3. Diffraction patterns of the $\mathrm{La}_{0.85} \mathrm{Ca}_{0.15} \mathrm{MnO}_{3}$ sample measured with DMC at $T=25 \mathrm{~K}$ and $250 \mathrm{~K}$. For $T=25 \mathrm{~K}$ the $y$ scale is shifted by 5000 counts. Miller indices highlight the most intense FM peaks.

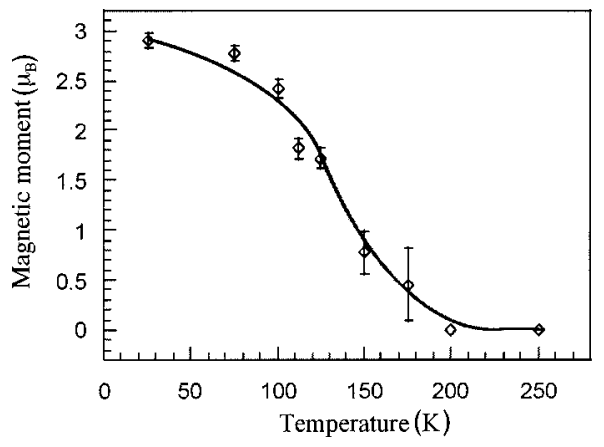

FIG. 4. Temperature dependence of the Mn magnetic moment (in Bohr magnetons) calculated from the neutron diffraction data (DMC). The line is a guide to the eye.

intensities of the nuclear reflections by magnetic contributions. The temperature of the ferromagnetic transition of about $T_{c}=170 \mathrm{~K}$, coincides well with the data of Schiffer et al. ${ }^{3}$ for the magnetic phase diagram of $\mathrm{La}_{1-x} \mathrm{Ca}_{x} \mathrm{MnO}_{3}$.

The refinements show best $\chi^{2}$ for Mn spins directed along the $c$ axis. However, the refined value of the Mn moment is not sensitive to the Mn-spin direction. The low temperature data obtained at HRFD show that the main magnetic contribution is to the (200) peak, which means at least that $\mu_{\mathrm{Mn}}$ is nonparallel to the $a$ axis. The temperature dependence of the ferromagnetic moment of $\mathrm{Mn}$ is shown in Fig. 4. The low temperature value of the magnetic moment is $\mu$ $=2.9(2) \mu_{B}$, and agrees well with Mn moments measured for the same compositions in Huang et al. ${ }^{4}$ It should be mentioned that this value is significantly less than an average total moment arising from a mixed $\mathrm{Mn}^{3+} / \mathrm{Mn}^{4+}$ lattice which amounts to $3.85 \mu_{B}$ for $15 \% \mathrm{Mn}^{4+}$.

\section{ELECTRON DIFFRACTION AND ELECTRON MICROSCOPY DATA}

In order to determine the actual symmetry selected area electron diffraction (SAED) and high-resolution electron microscopy (HREM) were performed using a Philips CM20 and a Jeol $4000 \mathrm{EX}$ instrument operating at $200 \mathrm{kV}$ and 400 $\mathrm{kV}$, respectively.

Most crystallites of $\mathrm{La}_{0.85} \mathrm{Ca}_{0.15} \mathrm{MnO}_{3}$ exhibit complicated electron diffraction patterns (EDP) where, because of twinning, two or more zone axis patterns are superimposed. Single SAED patterns were found occasionally; such patterns taken along the $[010]^{*},[100]^{*},[101]^{*}$, and [111]* zone axis are shown in Fig. 5 (the indexation is based on an orthorhombic unit cell with cell parameters $a=5.473 \AA$, $b$ $=7.745 \AA$, and $c=5.504 \AA$. The array of brightest spots on these patterns satisfy the extinction conditions imposed by the Pnma space group $(0 k l, k+l=2 n, h k 0, h=2 n)$. The presence of $00 l$ and $h 00$ reflections with $h, l \neq 2 n$ in the $[010]^{*}$ pattern is caused by double diffraction. However, the spots $0 k 0, k \neq 2 n$ are present in [101]*. A tilting experiment along $[0 k 0]^{*}$ reciprocal lattice row shows that these spots become hardly visible, but do not disappear completely, if the diffraction conditions do not allow these spots to be produced by double diffraction. Moreover, very weak $0 k l, k$ $+l \neq 2 n$ reflections were found in $[100]^{*}$ where no conditions for double diffraction exist. One might argue, however, 

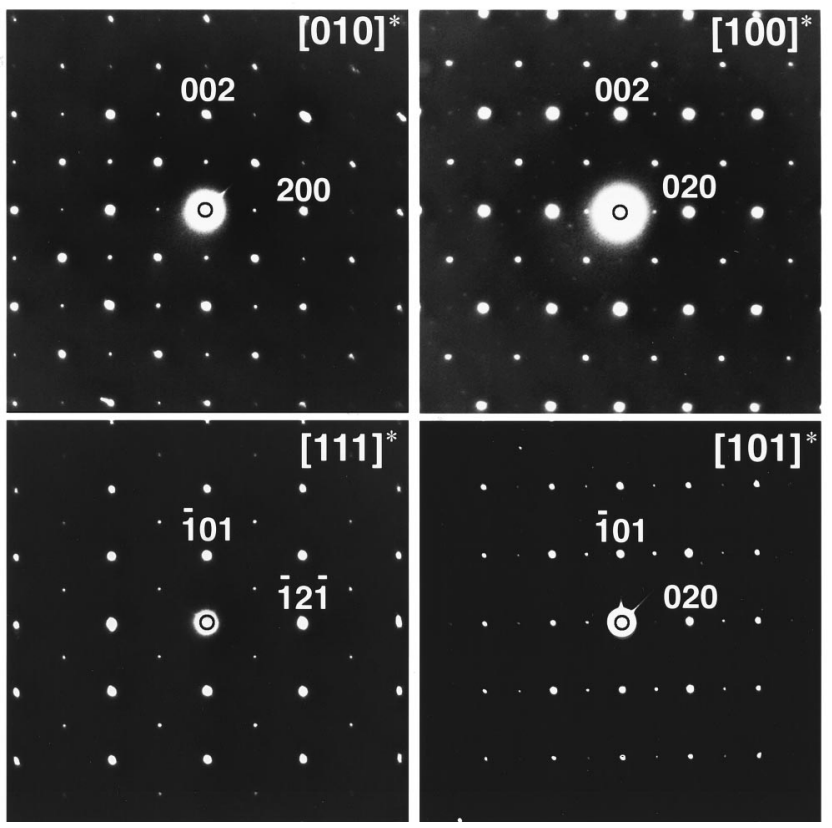

FIG. 5. Electron diffraction patterns taken along the [010]*, $[100]^{*},[101]^{*}$, and $[111]^{*}$ zone axes.

that these weak reflections could result from other orientation variants. The electron diffraction data provide an evidence of Pnma symmetry breaking; the weakness of additional reflections means that the atomic shifts leading to symmetry lowering are relatively small. The observed extinction conditions are consistent with either an orthorhombic $P m c 2_{1}$ or a monoclinic $P 2_{1} / c$ symmetry.

The [101] HREM image (Fig. 6) shows a square pattern of bright dots spaced by $3.8 \AA$. Rows of dots with different brightness alternate along [010] resulting in a $7.7 \AA$ periodicity. It has been verified that this effect is not introduced by crystal tilt or microscope misalignment. The $\mathrm{GdFeO}_{3}$-type perovskite structure, based on a Pnma space group, contains only single type of both $A$ and $\mathrm{Mn}$ cations and the projections of all $\mathrm{MnO}_{2}$ or $A \mathrm{O}$ layers along the [101] direction should exhibit an identical contrast. The $7.7 \AA$ " "period doubling" along [010] implies that two successive layers are no longer identical and, possibly, have a different geometry.

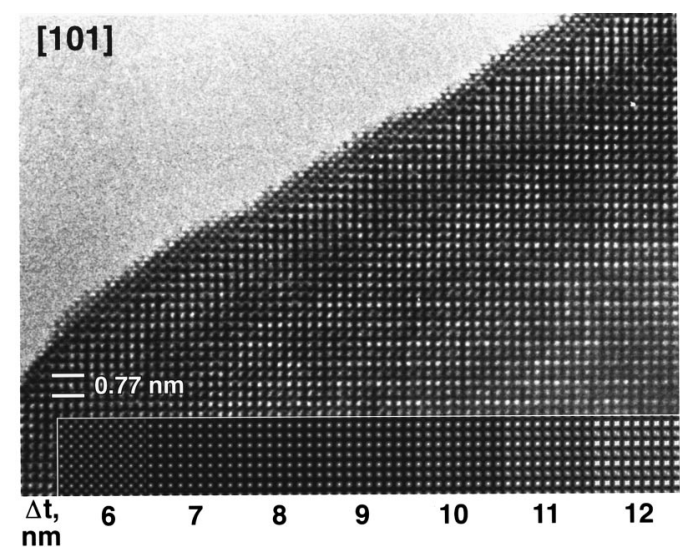

FIG. 6. HREM image along the [101] zone. The inset reproduces theoretical images calculated with $P 2{ }_{1} / c$ model $(\Delta f=$ $-60 \mathrm{~nm})$.
The difference in the brightness of the layers is almost invisible in the thin part of the crystallite (close to the crystal edge) and gradually increases with increasing thickness. We used the results of the neutron atomic structure refinement described below (Table I) to reproduce the features of the experimental image contrast on the calculated [101] HREM images. The calculations were made by means of the MacTempas software. It was found that calculations based on a $\mathrm{GdFeO}_{3}$-type structure with space group Pnma cannot provide a good correspondence between experimental and theoretical images at any thickness and defocus value. The correspondence was found to be satisfactory only when the monoclinic $P 2{ }_{1} / c$ model was used. The set of calculated images obtained at $\Delta f=-60 \mathrm{~nm}$ is shown in the inset in Fig. 6. It perfectly reproduces the intensity difference between successive layers as well as the variation of this difference with the thickness of the crystallite.

\section{ATOMIC STRUCTURE-NEUTRON DIFFRACTION DATA}

ED and HREM experiments reveal a violation of the Pnma symmetry. In order to determine precisely the origin of this phenomenon and to choose the correct model neutron powder diffraction (NPD) experiments were performed with a high resolution TOF Fourier diffractometer HRFD [ $d$-spacing range $(0.7-3.5) \AA, \delta d / d \approx 0.1 \%]$ at the IBR-2 pulsed reactor in Dubna for $10 \leqslant T \leqslant 290 \mathrm{~K}$. Rietveld refinements were carried out using the MRIA program over the $d$-spacing range $(0.8-2.5) \AA$.

It was found that the diffraction patterns could be rather well described in average by the standard structural model with the orthorhombic Pnma symmetry. In this approximation the temperature behavior of the average bond lengths and angles was found as typical: ${ }^{4,17}\langle\mathrm{Mn}-\mathrm{O}\rangle$ goes down with decreasing temperature and changes slope at $T_{c} ;\langle\mathrm{Mn}-\mathrm{O}-$ $\mathrm{Mn}\rangle$ also goes down until $T_{c}$, but then starts to increase (Fig. 7).

However in certain regions of the spectrum, especially around the (220)/(022) doublet, the agreement between the experimental and calculated pattern was unsatisfactory. Analysis showed that within the frame of the Pnma space group variation of any parameter including atom occupancy factors does not give an adequate description of this doublet. The general outlook of the diffraction pattern including the doublet region is only slightly affected by temperature variation (except for the appearance of a ferromagnetic component below $T_{c}$ ). Thus the fit was performed for the data acquired with the best statistical accuracy at $T=200 \mathrm{~K}$.

As electron diffraction indicates a violation of the extinction conditions for the Pnma space group, we suggested that the actual structure should be adequately described in one of its subgroups. Electron diffraction data are consistent with either $P m c 2_{1}$ or $P 2_{1} / c$ space groups. The former space group allows possibilities of splitting both $A$ or $B$ sites of the perovskitetype structure with a "layer-by-layer" ordered distribution in both cases. The $\mathrm{GdFeO}_{3}$-type structure (Pnma) is derived from the cubic perovskite by a combination of $\mathrm{MnO}_{6}$ octahedra tilts around $(110)_{c}$ and $(001)_{c}$ (Glazer notation $a^{+} b^{-} b^{-}$). ${ }^{18}$ Splitting of the cation positions due to a symmetry lowering from Pnma to $P m c 2_{1}$ is pos- 

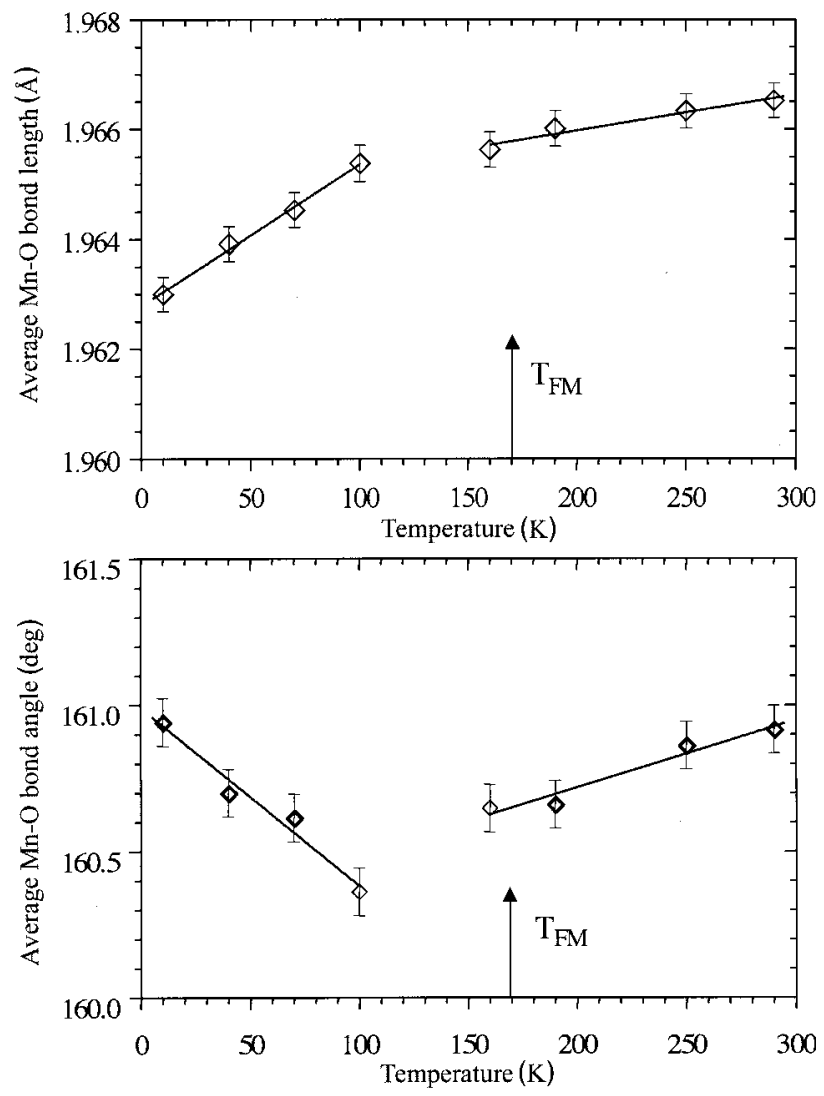

FIG. 7. Temperature dependence of average Mn-O distance and Mn-O-Mn bond angle, calculated in Pnma model. The lines are guides to the eye. Temperature of FM transition is shown by an arrow.

sible only if they are located on a $m$ mirror plane passing through the AO layers in the Pnma structure. Thus the groupsubgroup relations allow a splitting of the $A$ site only providing the tilt system remains unchanged. If we attempt to split the $B$ position only the $(001)_{c}$ tilting component is possible since the $(110)_{c}$ component is suppressed by the $m$ plane passing through the $\mathrm{MnO}_{2}$ layers. Nevertheless this possibility exists and was also verified by the Rietveld refinement using the NPD pattern.

With the octahedral tilt system $a^{+} b^{-} b^{-}$maintained, the splitting of the Mn position inevitably leads to a monoclinic distortion of the crystal structure. Mn atoms may adopt different arrangements; the possibility consistent with the SAED data corresponds to a $P 2_{1} / c$ space group with a "layer-by-layer" ordering of the Mn atoms.

The three possibilities (two models for $P m c 2_{1}$ and one model for $P 2{ }_{1} / c$ ) were verified by the Rietveld refinement. The refinement of the $P m c 2_{1}$ model with two $A$-cation sites yielded reliability factors $\left(\chi^{2}=1.58, R_{P}=0.116, \quad R_{w}\right.$ $=0.078$ ), atomic positions, and interatomic distances almost identical with those for the Pnma model. The refinement is not sensitive to the relative occupation of nonequivalent $A$ sites by $\mathrm{La}$ and $\mathrm{Ca}$ in spite of a significant difference in their atomic scattering factors $(0.824$ and 0.490 for $\mathrm{La}$ and $\mathrm{Ca}$, respectively). The variation of the occupation factors does not provide a better profile description or a $\chi^{2}$ value lowering. On the other hand, the $P m c 2_{1}$ model with two Mn sites gives a satisfactory description of the $(220) /(022)$ doublet,

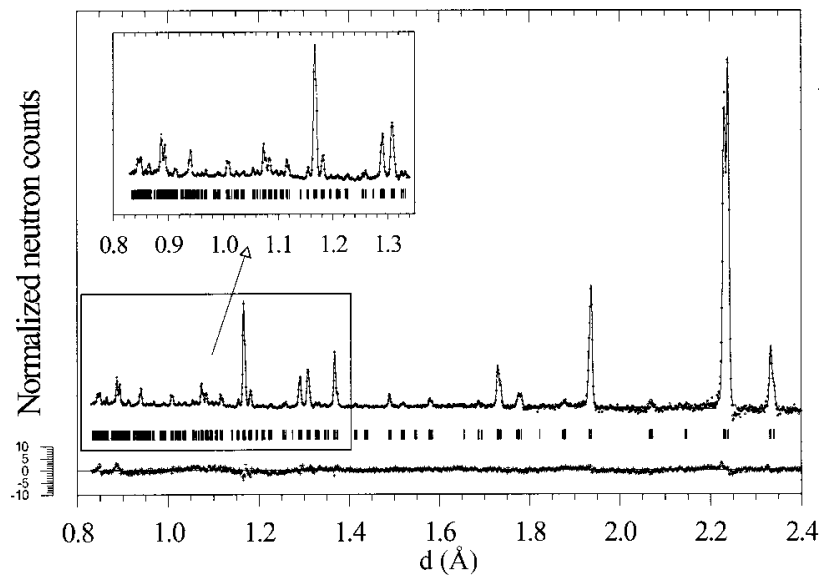

FIG. 8. Neutron diffraction pattern of $\mathrm{La}_{0.85} \mathrm{Ca}_{0.15} \mathrm{MnO}_{3}$ sample, measured at $200 \mathrm{~K}$ with the HRFD diffractometer. Experimental, calculated $\left(P 2{ }_{1} / c\right.$ space group), and difference curves are shown. The difference curve is normalized on the mean square deviation.

but the general fit is much worse compared to the Pnma model $\left(\chi^{2}=3.21, R_{P}=0.139, R_{w}=0.111\right)$.

The refinement in the $P 2{ }_{1} / c$ space group leads to a significantly better fit of the experimental neutron diffraction pattern $\left(\chi^{2}=1.43, R_{P}=0.107, R_{w}=0.074\right)$. The main difference with the Pnma case arises from a much more accurate fit of the doublet. The example of the Rietveld refinement for the $P 2_{1} / c$ model is shown in Fig. 8. The fitting of the pattern in the $d$-spacing interval around the $(220) /(022)$ orthorhombic doublet for the two structure models is illustrated in Fig. 9. The refinement of the occupation factors did not reveal any deviation from the full stoichiometry within the error limits, in agreement with the chemical analysis data. The final refinement was carried out with a fixed full occupancy for the atomic positions. The comparison of the refined structural parameters with the parent Pnma model is shown in Table I; most relevant interatomic distances and bond angles for the $P 2_{1} / c$ model are listed in Table II.

A two phase Pnma model as another possibility for a better fitting of the high-resolution neutron diffraction pattern gave reliability factors very close to those of the $P 2{ }_{1} / c$ model and could not be rejected on the basis of a neutron diffraction data. The refinement of this model was performed with the positional parameters for two phases forced to coincide and yielded reliability factors comparable with the $P 2{ }_{1} / c$ model $\left(\chi^{2}=1.47, R_{P}=0.110, R_{w}=0.075\right)$. Refined lattice parameters for the two phases are $a=5.4764(1) \AA$, $b=7.7488(2) \AA, c=5.5036(2) \AA$ and $a=5.4680(2) \AA, b$ $=7.7383(4) \AA, c=5.5058(4) \AA$, respectively. The ratio of phase volume fractions is approximately $2: 1$. However, this possibility does not account for ED and HREM data available. Thus we may conclude that the actual symmetry is monoclinic $P 2_{1} / c$. The neutron diffraction pattern has the general outlook close to that for the Pnma model, but some features are reproduced only if ordering in the $B$ sublattice is taken into account.

The temperature dependencies of the lattice parameters and the cell volume for the $P 2_{1} / c$ symmetry are shown in Fig. 10. A very small but clearly seen cell volume discontinuity occurs at a temperature just below $T_{C}$. The volume jump in this compound is about $0.035 \%$ which is nearly four 

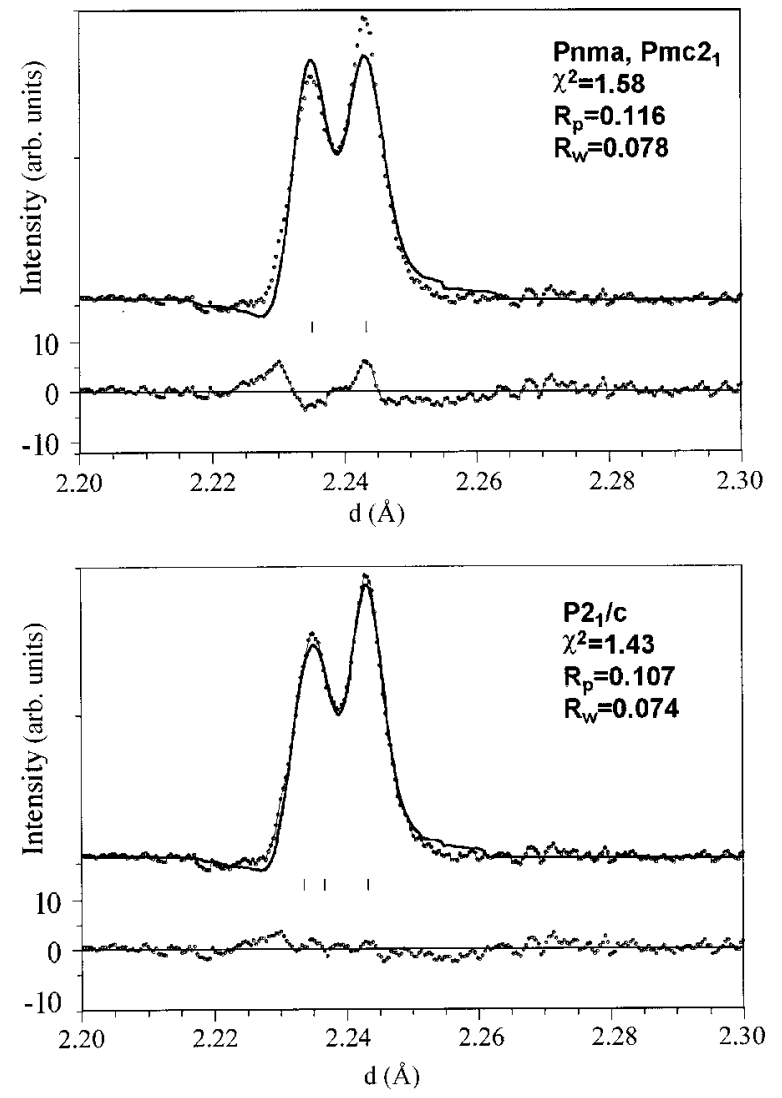

FIG. 9. A part of $\mathrm{La}_{0.85} \mathrm{Ca}_{0.15} \mathrm{MnO}_{3}$ diffraction pattern around the (220)/(022) doublet refined in Pnma (top) and $P 2{ }_{1} / c$ (bottom) models.

times smaller than was found for $\mathrm{La}_{075} \mathrm{Ca}_{0.25} \mathrm{MnO}_{3},{ }^{19}$ or for $\mathrm{La}_{0.35} \mathrm{Pr}_{0.35} \mathrm{Ca}_{0.30} \mathrm{MnO}_{3},{ }^{20}$ but still more than 100 times larger than the typical value for conventional FM crystals.

\section{MAGNETIC DISORDER- $\mu$ SR DATA}

To get more insight into the $\mathrm{La}_{0.85} \mathrm{Ca}_{0.15} \mathrm{MnO}_{3}$ magnetic structure muon spin relaxation $(\mu \mathrm{SR})$ experiments were carried out at PSI (Villigen) on the GPD spectrometer at the $\mu E 4$ muon beam line, using high energy muons (70 MeV/c). A powder sample of $\mathrm{La}_{0.85} \mathrm{Ca}_{0.15} \mathrm{MnO}_{3}$ was pressed into a round pellet with a $19 \mathrm{~mm}$ diameter and $8 \mathrm{~mm}$ height. Measurements were performed in zero (ZF) and transverse to the initial muon spin polarization (TF) external magnetic field. The pellet was glued to the silver holder which was mounted on the cold finger of the closed cycle helium refrigerator. The external magnetic field was applied perpendicular to the pellet axis.

The $\mu \mathrm{SR}$ data show that the local spin configuration has a high extent of disorder because no precession or detectable fast relaxing signals were detected in the zero field experiment at low temperatures below $T_{C}$. It means that the local magnetic field on the muon produced by Mn spins below $T_{C}$ has an extremely wide distribution, $\left\langle\Delta B^{2}\right\rangle \approx\left\langle B^{2}\right\rangle$, thus the signal is strongly damped resulting in the loss of polarization. However, a transition to a magnetically ordered state has been detected in the TF- $\mu$ SR experiment by decrease in muon fraction precessing with the external magnetic field frequency. The transition temperature determined in this ex-
TABLE I. Comparison of Pnma and $P 2{ }_{l} / c$ models refined from neutron diffraction data $(T=200 \mathrm{~K})$.

\begin{tabular}{lll}
\hline \hline Space group & \multicolumn{1}{c}{ Pama } & \multicolumn{1}{c}{$P{ }_{l} / c$} \\
\hline$a, \AA$ & $5.47332(8)$ & $7.74476(6)$ \\
$b, \AA$ & $7.74525(13)$ & $5.50398(4)$ \\
$c, \AA$ & $5.50387(8)$ & $5.47351(4)$ \\
$\beta, \mathrm{deg}$ & 90 & $90.091(2)$ \\
$0.85 \mathrm{La}+0.15 \mathrm{Ca}$ & $\mathbf{4 c}$ & $\mathbf{4 e}$ \\
$x / a$ & $0.0190(4)$ & $0.2483(6)$ \\
$y / b$ & $1 / 4$ & $-0.0033(3)$ \\
$z / c$ & $-0.0034(4)$ & $-0.0174(3)$ \\
$\mathrm{Mn} 1$ & $\mathbf{4 b},(0,0,1 / 2)$ & $\mathbf{2 c}(0,1,1 / 2)$ \\
$\mathrm{Mn} 2$ & & $\mathbf{2 d}(1 / 2,0,1 / 2)$ \\
$\mathrm{O} 1$ & $\mathbf{4 c}$ & $\mathbf{4 e}$ \\
$x / a$ & $0.4991(8)$ & $0.2465(9)$ \\
$y / b$ & $1 / 4$ & $0.0637(2)$ \\
$z / c$ & $0.0637(4)$ & $0.5013(5)$ \\
$\mathrm{O} 2$ & $\mathbf{8 d}$ & $\mathbf{4 e}$ \\
$x / a$ & $0.2712(4)$ & $0.0315(2)$ \\
$y / b$ & $0.0327(2)$ & $0.7363(5)$ \\
$z / c$ & $0.7326(5)$ & $0.7230(4)$ \\
$\mathrm{O} 3$ & & $\mathbf{4 e}$ \\
$x / a$ & & $0.4671(2)$ \\
$y / b$ & & $-0.2253(5)$ \\
$z / c$ & & $0.2319(4)$ \\
$\chi^{2}, R_{p}, R_{w}$ & $1.58,0.116,0.078$ & $1.43,0.107,0.074$ \\
\hline \hline
\end{tabular}

periment scales well with the magnetic susceptibility and the neutron diffraction data.

In the $\mu \mathrm{SR}$ experiments ${ }^{21}$ performed on $\mathrm{La}_{0.67} \mathrm{Sr}_{0.33} \mathrm{MnO}_{3}$ strong damping of the signal was also observed; the linewidth measured was roughly consistent with an incoherent mixture of $\mathrm{Mn}^{+3} / \mathrm{Mn}^{+4}$ and the calculated distribution of demagnetization factors arising from varying grain shapes. Moreover, the presence of a $\mathrm{Mn}^{+3} / \mathrm{Mn}^{+4}$ mixture causes structural disorder as the PDF analysis shown. ${ }^{22}$ However, in the present case it is not connected with a nonuniform cation distribution or vacancy clustering as follows from EDX and chemical analysis. The reduced value of the ordered magnetic moments of $\mathrm{Mn}$ determined from neutron diffraction $2.9 \mu_{B}$ ) evidences the existence of spatial fluctuations of the Mn spin direction leading to a further increase of the local field value dispersion.

TABLE II. Selected interatomic distances $(\AA)$ and angles (deg) for the Pnma and $P 2_{l} / c$ models $(T=200 \mathrm{~K})$.

\begin{tabular}{lr}
\hline \hline Pnma & $P 2{ }_{l} / c$ \\
Mn-O1 $2 \times 1.9678(4)$ & Mn1-O1 $2 \times 1.941(7)$ \\
Mn-O2 $2 \times 1.949(3)$ & Mn1-O2 $2 \times 1.912(3)$ \\
$2 \times 1.976(2)$ & $2 \times 2.013(2)$ \\
& Mn2-O1 $2 \times 1.994(7)$ \\
& Mn2-O3 $2 \times 1.938(2)$ \\
Mn-O1-Mn 159.5(2) & $2 \times 1.991(3)$ \\
Mn-O2-Mn 162.8(2) & Mn1-O1-Mn2 159.5(3) \\
& Mn1-O2-Mn1 162.9(1) \\
& Mn2-O3-Mn2 162.2(1) \\
\hline
\end{tabular}



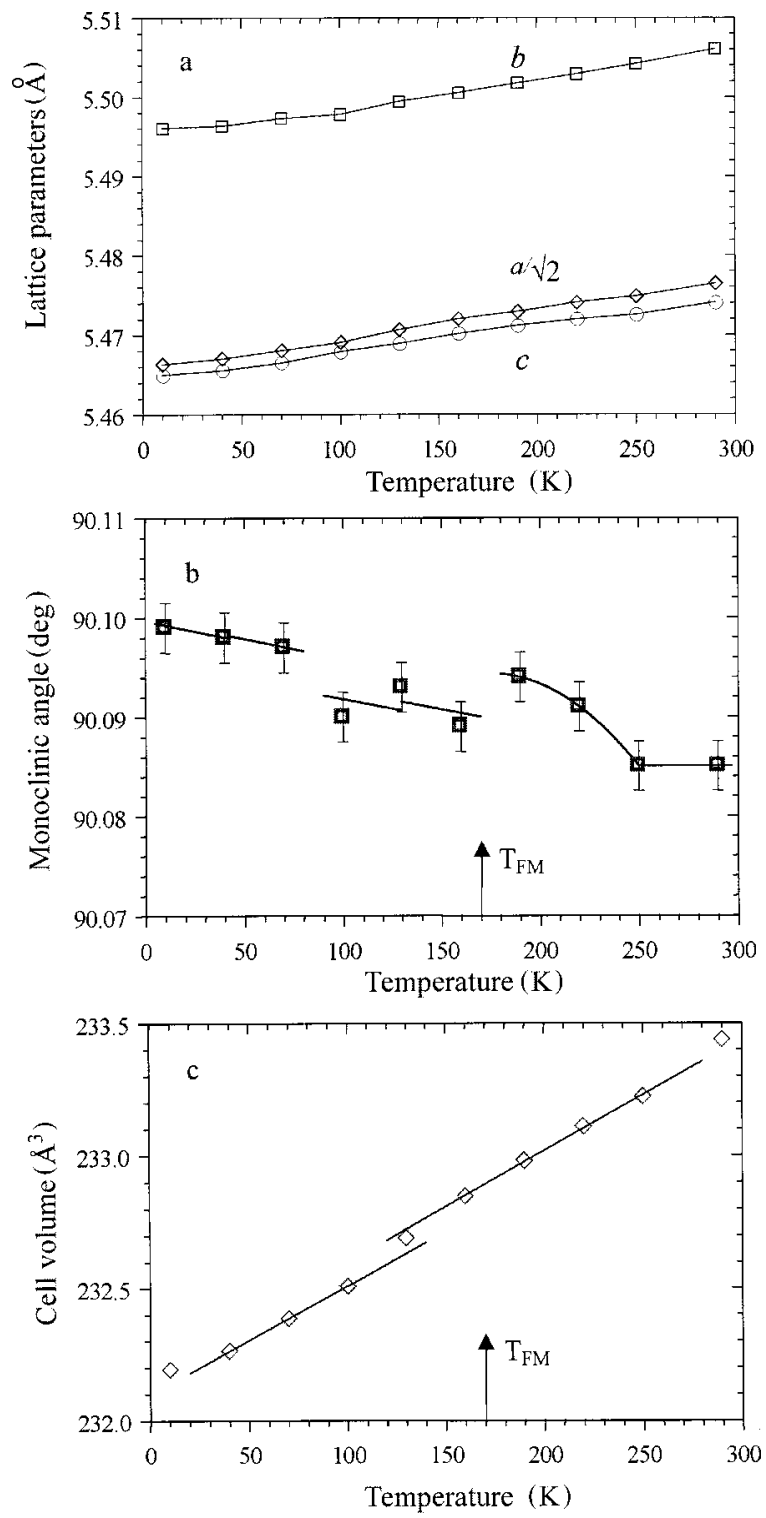

FIG. 10. Temperature dependencies of lattice parameters (a), monoclinic angle (b), and cell volume (c) after $\mathrm{La}_{0.85} \mathrm{Ca}_{0.15} \mathrm{MnO}_{3}$ refinement in the frame of $P 2_{1} / c$ space group. For this setting $a_{m}$ $\approx b_{0} \approx 2 a_{\mathrm{per}}, \quad b_{m} \approx c_{0} \approx \sqrt{ } 2 a_{\mathrm{per}}, \quad$ and $c_{m} \approx a_{0} \approx \sqrt{ } 2 a_{\mathrm{per}}$, where $(a, b, c)_{m}$ are monoclinic, $(a, b, c)_{0}$ are orthorhombic, and $(a, b, c)_{\text {per }}$ are perovskite cell constants. Lines at (b) are guides to the eye. Lines at (c) are linear fits for $40 \leqslant T \leqslant 100 \mathrm{~K}$ and $160 \leqslant T$ $\leqslant 240 \mathrm{~K}$. Temperature of FM transition is shown by arrows.

\section{DISCUSSION}

We performed a detailed crystal structure investigation for the $\mathrm{La}_{0.85} \mathrm{Ca}_{0.15} \mathrm{MnO}_{3}$ composition by a combination of electron diffraction, high resolution electron microscopy, and neutron diffraction. Various possible crystal structure models allowed by ED were verified by Rietveld refinement of the NPD data. On the basis of the entire set of data we claim that the actual crystal structure is monoclinic $P 2{ }_{1} / c$.

The schematic representation of the structural evolution upon the symmetry lowering from Pnma to Pmc $2_{1}$ (two $A$-cation sites) or $P 2{ }_{1} / c$ (two Mn sites) is presented on Fig. 11. The scheme of Mn-O bond arrangements and corresponding orbital ordering for the $P 2_{1} / c$ case is shown in

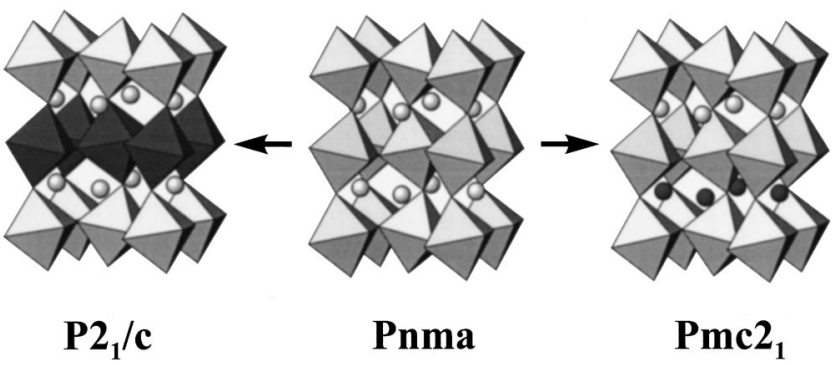

FIG. 11. Schematic representation of cation ordering corresponding to $P m c 2_{1}$ and $P 2_{1} / c$ models. Nonequivalent positions for $A$ cations in the $P m c 2_{1}$ model and nonequivalent $\mathrm{MnO}_{6}$ octahedra in the $P 2_{1} / c$ one are shown as light and dark ones.

Fig. 12. Within this model two nonequivalent Mn sites have significantly different oxygen environment, the $\mathrm{Mn}^{1 \mathrm{O}_{6}}$ octahedra being "apically elongated" (two long and four short

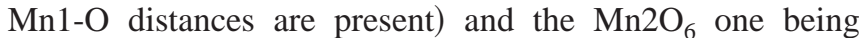
" 'apically compressed"' (two short and four long Mn2-O distances). The "apically elongated" Jahn-Teller distortion is the most common case and it was reported in particular for undoped $\mathrm{RMnO}_{3}$ perovskites. ${ }^{23,24}$ "Apically compressed",
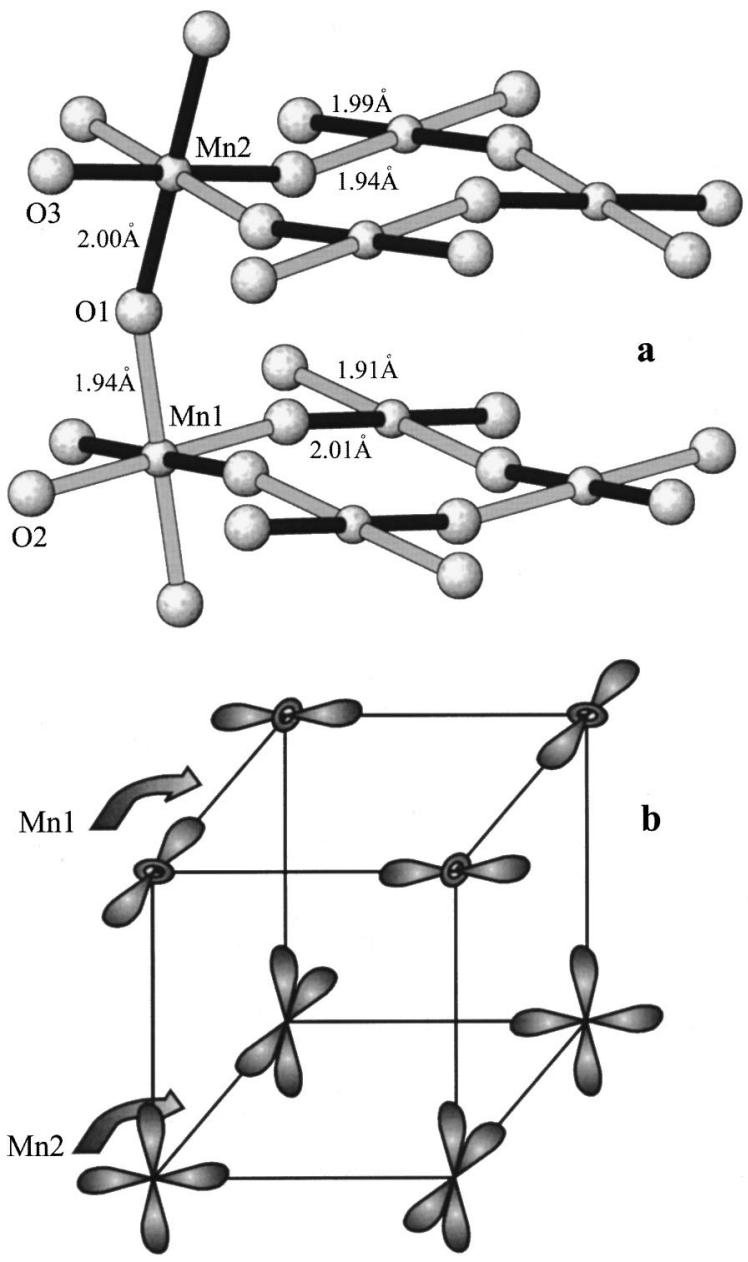

FIG. 12. (Top): Scheme of Mn-O bonds arrangement for the $P 2_{1} / c$ model. Long and short $\mathrm{Mn}-\mathrm{O}$ bonds are shown as filled and empty, respectively. (Bottom): Orbital ordering scheme. $d_{z 2}$-type orbitals are shown for the "apically elongated" octahedra and $d_{x 2-y 2}$-type orbitals for the "apically compressed" ones. 
distortion is a more rare possibility found for instance in cation-deficient $\mathrm{La}_{0.9} \mathrm{MnO}_{3}$ (Ref. 25) and charge-ordered $\mathrm{La}_{0.5} \mathrm{Ca}_{0.5} \mathrm{MnO}_{3}{ }^{26}$ Undoped $R \mathrm{MnO}_{3}$ compounds exhibit $d$-type orbital ordering (corresponding to the so-called $\mathrm{O}^{\prime}$ structure) with the long axes of the Jahn-Teller distorted octahedra in two successive $\left(\mathrm{MnO}_{2}\right)$ layers in the (010) direction connected by a mirror plane $y=1 / 4$ (all designations refer to Pnma). In this case no symmetry lowering is required for the description of the ordered state; this becomes necessary if two Mn sites are no longer equivalent.

The estimate of local modes characterizing the degree of Jahn-Teller distortion defined as $Q_{2}=2(1-s) / \sqrt{ } 2$ and $Q_{3}$ $=2(2 m-1-s) / \sqrt{6},{ }^{27}$ where $l, m$, and $s$ stand for long, medium, and short Mn-O distances, respectively, yields $Q_{2}$ $=0.143(1), Q_{3}=-0.035(7)$ for the $\mathrm{Mn}_{10}$ octahedron and $Q_{2}=0.079(7), Q_{3}=0.041(2)$ for the $\mathrm{Mn}_{2} \mathrm{O}_{6}$ one; positive and negative values for the $Q_{3}$ mode correspond to an elongation or a compression of the octahedra along the $d_{z^{2}}$-type orbital direction. Corresponding values for the parent Pnma structure scale to $Q_{2}=0.038(1), Q_{3}=0.009(3)$, so the symmetry lowering is connected with the development of a static Jahn-Teller effect.

The different oxygen environment for two nonequivalent Mn atoms suggests the possibility of a structural distortion as a consequence of charge ordering. For the elucidation of this possibility bond valence sum (BVS) calculations were performed using Mn-O distances obtained from NPD data refinement. The Mn valence $\left(V_{\mathrm{Mn}}\right)$ was calculated as a sum over the first coordination sphere of bond valences as $\exp \left[\left(R_{0}-R_{1}\right) / B\right]$, where $R_{0}=1.759 \AA$ (weighed average of values $1.753 \AA$ for $\mathrm{Mn}^{+4}$ and $1.760 \AA$ for $\mathrm{Mn}^{+3}$ ) and $B$ $=0.37 .^{28}$ The calculations revealed $V_{\mathrm{Mn} 1}=3.55, V_{\mathrm{Mn} 2}$ $=3.36$. The absolute values are substantially overestimated as is typical for cations exhibiting a strong Jahn-Teller effect. ${ }^{29}$ Similar results were obtained for $\mathrm{SrMnO}_{3-\delta}$ with a composition close to stoichiometric, and instead we used $R_{0}=1.725 \AA$, which was proven to give reliable results for the latter compound. The obtained values were $V_{\mathrm{Mn} 1}=3.24$ and $V_{\mathrm{Mn} 2}=3.06$, indicative of a possible charge ordering. Thus one may treat the Mn2 site as occupied by Mn(III) only and $\mathrm{Mn} 1$ site as occupied commonly by $\mathrm{Mn}$ (III) and $\mathrm{Mn}$ (IV) and we propose charge ordering as a microscopic reason for the splitting of the $B$ site and consequently the symmetry lowering.

The ordered placement of Mn atoms can lead to a profound modification of the physical properties. Since ordering has a two-dimensional character, it would not necessarily lead to an insulating behavior. Nevertheless, the existence of different Mn-O-Mn angles (see Table II) may lead to a complicated pattern of exchange interactions, different from those expected from the Pnma model.

The origin of the upturn in the resistivity vs temperature curve is not unambiguous. The appearance of a similar upturn in samples with $\mathrm{La}_{0.9} \mathrm{Sr}_{0.1} \mathrm{MnO}_{3}$ and $\mathrm{La}_{0.85} \mathrm{Sr}_{0.15} \mathrm{MnO}_{3}$ compositions was connected ${ }^{3}$ with the formation of a "polaron ordered" phase. This phase, as described by Yamada et al. ${ }^{3}$ has also nonequivalent oxygen surrounding for $\mathrm{Mn}$ atoms in successive layers, but has an additional ordering within layers leading to a localization of the holes. Phase separation with charge carriers localized within the FM droplets is hardly possible since the sample was characterized as purely FM. Such a behavior can also be a result of weak localization ${ }^{30}$ since a high extent of disorder was detected by $\mu \mathrm{SR}$ and the resistivity value is close to Mott's maximum. A similar $\left(T_{\min } \sim 15 \mathrm{~K}\right)$ behavior in $\left(\mathrm{Pr}_{0.1} \mathrm{Ce}_{0.4} \mathrm{Sr}_{0.5}\right) \mathrm{MnO}_{3}$ was attributed $^{31}$ to a Kondo-like effect. Since no structural data are available in this temperature range we cannot say definitely whether such a behavior has a structural origin or not.

One should also note that most probably the observed monoclinic distortion is not only confined to this single concentration. A monoclinic symmetry was also observed by TEM in different CMR thin films ${ }^{11}$ as well as in bulk manganites. ${ }^{12}$ Similar distortions are expected to occur here also. The observed monoclinic distortion is stable around $x$ $=0.125$, a point where polaron ordering of the type proposed by Yamada ${ }^{8}$ sets in. This transition to the "Yamada" state would probably be similarly represented as a valence ordering as described in our model. It is not clear however at which doping level this ordering will be most pronounced. Samples are being prepared to investigate this effect.

\section{ACKNOWLEDGMENTS}

The authors are grateful to K. Kalmykov for carefully performed EDX analysis, P. E. Kazin for magnetic susceptibility measurements, A. D. Bozhko and V. A. Bogoyavlenskiy for resistivity measurements, and A. A. Bosak for the help with chemical analysis. We are also grateful to D. I. Khomskii for helpful discussions. A. Abakumov and O. Lebedev thank DWTC and FWO (Belgium), respectively, for financial support during their stay at the University of Antwerp. This work has been performed within the framework of IUAP 4/10 and was supported by Russian Science Foundation (Grants No. 97-02-16665 and No. 98-15-96058).
*On leave from Institute of Crystallography, RAS, Leninsky pr. 59, 117333 Moscow, Russia.

${ }^{\dagger}$ Author to whom correspondence should be addressed.

${ }^{1}$ R. von Helmolt, J. Wecker, B. Holzapfel, L. Schultz, and K. Samwer, Phys. Rev. Lett. 71, 2331 (1993).

${ }^{2}$ J. B. Goodenough, Phys. Rev. 100, 564 (1955).

${ }^{3}$ P. Schiffer, A. P. Ramirez, W. Bao, and S.-W. Cheong, Phys. Rev. Lett. 75, 3336 (1995).

${ }^{4}$ Q. Huang, A. Santoro, J. W. Lynn, R. W. Erwin, J. A. Borchers, J. L. Peng, K. Ghosh, and R. L. Greene, Phys. Rev. B 58, 2684 (1998).

${ }^{5}$ C. Zener, Phys. Rev. 82, 403 (1951).
${ }^{6}$ E. L. Nagaev, Phys. Usp. 38, 497 (1995).

${ }^{7}$ H. Kawano, R. Kajimoto, M. Kubota, and H. Yoshizawa, Phys. Rev. B 53, R14 709 (1996).

${ }^{8}$ Y. Yamada, O. Hino, S. Nohdo, R. Kanao, T. Inami, and S. Katano, Phys. Rev. Lett. 77, 904 (1996).

${ }^{9}$ Y. Endoh, K. Hirota, S. Ishibara, S. Okamoto, Y. Murakami, A. Nishizawa, T. Fukuda, H. Kimura, H. Nojiri, K. Kaneko, and S. Maekawa, Phys. Rev. Lett. 82, 4328 (1999).

${ }^{10}$ D. E. Cox, T. Iglesias, G. Shirane, K. Hirota, and Y. Endoh, Powder Diffr. 14, 147 (1999).

${ }^{11}$ O. I. Lebedev, G. Van Tendeloo, S. Amelinckx, B. Leibold, and H.-U. Habermeier, Phys. Rev. B 58, 8065 (1998). 
${ }^{12}$ M. Hervieu, G. Van Tendeloo, V. Caignaert, A. Maignan, and B. Raveau, Phys. Rev. B 53, 14274 (1996).

${ }^{13}$ C. H. Chen, S.-W. Cheong, and H. Y. Hwang, J. Appl. Phys. 81, 4326 (1997).

${ }^{14}$ S. Mori, C. H. Chen, and S.-W. Cheong, Nature (London) 392, 473 (1998).

${ }^{15}$ P. G. Radaelli, D. E. Cox, L. Capogna, S.-W. Cheong, and M. Marezio, Phys. Rev. B 59, 14440 (1999).

${ }^{16}$ S. N. Mudretsova, A. F. Maiorova, A. A. Bosak, O. Yu. Gorbenko, A. R. Kaul, N. A. Babushkina, and L. M. Belova, Zhurnal Fizicheskoj Himii (Russian) (to be published).

${ }^{17}$ P. G. Radaell, G. Iannone, M. Marezio, H. Y. Hwang, S.-W. Cheong, J. D. Jorgensen, and D. N. Argyriou, Phys. Rev. B 56, 8265 (1997).

${ }^{18}$ A. M. Glazer, Acta Crystallogr., Sect. B: Struct. Crystallogr. Cryst. Chem. 28, 3384 (1972).

${ }^{19}$ P. G. Radaelli, M. Marezio, H. Y. Hwang, and S.-W. Cheong, J. Solid State Chem. 122, 444 (1996).

${ }^{20}$ A. M. Balagurov, V. Yu. Pomjakushin, V. L. Aksenov, N. M. Plakida, N. A. Babushkina, L. M. Belova, O. Yu. Gorbenko, A. R. Kaul, P. Fischer, M. Gutmann, and L. Keller, Pis'ma Zh. Eksp. Teor. Fiz. 67, 672 (1998) [JETP Lett. 67, 705 (1998)].

${ }^{21}$ R. H. Heffner, L. P. Le, M. F. Hundley, J. J. Neumeier,
G. M. Luke, K. Kojima, B. Nachumi, Y. J. Uemura, D. E. MacLaughlin, and S.-W. Cheong, Phys. Rev. Lett. 77, 1869 (1996).

${ }^{22}$ S. J. L. Billinge, R. G. DiFrancesco, G. GH. Kwei, J. J. Neumeier, and J. D. Thompson, Phys. Rev. Lett. 77, 715 (1996).

${ }^{23}$ A. A. Elemans, B. Van Laar, K. R. Van der Veen, and B. O. Loopstra, J. Solid State Chem. 3, 238 (1971).

${ }^{24}$ P. Norby, I. G. Krogh Andersen, E. Krogh Andersen, and N. H. Andersen, J. Solid State Chem. 119, 191 (1995).

${ }^{25}$ A. Maignan, C. Michel, M. Hervieu, and B. Raveau, Solid State Commun. 101, 277 (1997).

${ }^{26}$ P. G. Radaelli, D. E. Cox, M. Marezio, S.-W. Cheong, P. E. Schiffer, and A. P. Ramirez, Phys. Rev. Lett. 75, 4488 (1995).

${ }^{27}$ J. Kanamori, J. Appl. Phys. 31, 14S (1960).

${ }^{28}$ I. D. Brown and D. Altermatt, Acta Crystallogr., Sect. B: Struct. Sci. 41, 244 (1985).

${ }^{29}$ A. Salinas-Sanchez, J. L. Garcia-Munoz, J. Rodriguez-Carvajal, R. Saez-Puche, and J. L. Martinez, J. Solid State Chem. 100, 201 (1992).

${ }^{30}$ P. A. Lee and T. V. Ramakrishnan, Rev. Mod. Phys. 57, 287 (1985).

${ }^{31}$ A. Sundaresan, V. Caignaert, A. Maignan, B. Raveau, and E. Suard, Phys. Rev. B 60, 533 (1999). 